\title{
Construyendo "la identidad del excluido": Etnografía del aprendizaje situado de los niños en una escuela básica municipal de Chile*
}

\author{
Building up "the identity of the excluded": Ethnography of children's \\ situated learning in a Chilean public primary school \\ Construindo a identidade do excluído: Etnografia do aprendizado situado \\ de crianças em uma escola básica municipal do Chile
}

\section{Laura Luna Figueroa}

Pontificia Universidad Católica de Chile Campus Villarrica, Villarrica, Chile. Teléfono: (52) 45 2413693. Correo electrónico: 1luna@uc.cl

\section{RESUMEN}

Basado en una etnografía escolar de un establecimiento municipal de enseñanza básica de la Araucanía, este artículo analiza la participación y las experiencias de los estudiantes de un VI año durante el desenvolvimiento de las clases. Desde una perspectiva que considera el aprendizaje como una forma de participación en una comunidad de práctica se analizan las formas de resistencia que los estudiantes desarrollan durante las actividades didácticas como una reacción a prácticas pedagógicas inadecuadas y excluyentes. Estas a su vez son, en buena medida, el producto de un sistema de rendición de cuenta que desprofesionaliza la labor docente y contribuye a su falta de motivación hacia la enseñanza. Se argumenta que, en este marco, la participación de los estudiantes en el aula termina configurando una forma de ser y de hacer que no favorece una positiva incorporación de los niños a la sociedad y a la vida ciudadana.

Palabras clave: etnografía escolar, aprendizaje situado, identidad, niños, accountability.

\section{ABSTRACT}

Based on a school ethnography of a public primary school of Araucanía Region, this article analyses sixth grade students' participation and experiences during class time. From a perspective that holds learning as a form of participation in a community of practice, it analyses the forms of resistance that students develop during academic activities as a reaction against exclusive and inadequate pedagogical practices. The latter are, to a fair degree, the product of an accountability system which undermines teachers' professionalism and contributes to their lack of motivation towards teaching. In this context, it is argued that student's classroom participation ends up shaping up a way of being and doing that does not promote a positive incorporation of children into civic society and life.

Key words: school ethnography, situated learning, identity, children, accountability.

\section{RESUMO}

Baseado em uma etnografia escolar de um estabelecimento municipal de Ensino Básico municipal da região de Araucanía, analisa-se a participação e a experiência dos estudantes de um sexto ano durante o desenvolvimento das aulas. Desde uma perspectiva que considera o aprendizado como uma forma de participação em uma comunidade de prática, analisam-se as formas de resistência que os estudantes desenvolvem ao longo das atividades didáticas como reação às práticas pedagógicas inadequadas e excludentes. Estas, por sua vez e em boa

Proyecto Fondecyt N ${ }^{\circ} 11110390$. 
medida, são produto de um sistema de prestação de contas que desprofissionaliza o trabalho docente e contribui para a falta de motivação para o ensino. Argumenta-se que, nesse cenário, a participação dos estudantes nas aulas acaba configurando uma forma de ser e de fazer que desfavorece a incorporação positiva das crianças na sociedade e na vida cidadã.

Palavras chave: etnografia escolar, aprendizado situado, identidade, crianças, prestação de contas.

\section{INTRODUCCIÓN}

La literatura reciente ha generado un panorama exhaustivo del sistema educativo chileno. Al igual que otros países de Latinoamérica y en sintonía con una tendencia mundial (Ball, 1998; Falabella, 2007; Falabella y Opazo, 2014), Chile ha consolidado un modelo que intenta combinar una racionalidad neoliberal basada en las ideas de libertad de elección, eficiencia, esfuerzo y responsabilidad que promueven la privatización, con otra que mantiene las ventajas de un fuerte control estatal para asegurar estándares a nivel nacional; trasparentar la oferta de mercado mediante un sistema centralizado de información a la población (SIMCE) y, al mismo tiempo, generar estrategias en búsqueda de la igualdad de oportunidades e inclusión social, como es la entrega de recursos a las escuelas consideradas en desventaja socio-económica por medio de la ley SEP (Bellei, 2007; Assaél et al., 2011; Corvalán, 2013).

Los efectos de las políticas públicas sobre la calidad de la educación han sido discutidos en relación a una variedad de aspectos: las dinámicas sociales relacionadas, como la segmentación social (Valenzuela et al., 2010; Valenzuela et al., 2013), las problemáticas a nivel organizacional y de gestión escolar (Astudillo \& Imbarack, 2013; Falabella \& Opazo, 2014), las prácticas y subjetividad de los docentes (Acuña et al., 2014; Falabella \& Opazo, 2014), los mecanismos y criterios de decisión de los padres (Carrasco \& Martín, 2012), entre otros. Sin embargo, son aún escasos los estudios sobre las experiencias de los miembros de las comunidades escolares en el marco de las políticas de rendición de cuenta, estandarización y selección, pese a que, tal como sostiene Falabella (2014) la instalación de la "escuela performativa" a nivel global ha llevado a una profunda transformación ética en la manera en que la escolarización es entendida y vivida" (Falabella, 2014: 15); los trabajos de Ball (2003) y Niesche (2015) son excelentes ejemplos de este fenómeno. En esta línea, Falabella y Opazo (2014), ponen en evidencia las dinámicas que gatilla entre los profesores el sistema de aseguramiento de la calidad de la educación. Los docentes orientan su quehacer en función de una "gestión de la demostración" del desempeño y del conseguimiento de logros en perjuicio de una labor educativa en el ámbito de desarrollo personal de los niños y de la convivencia así como en desmedro de la innovación pedagógica, lo que se convierte también en fuente de conflicto y tensión para ellos. Por otra parte, este estudio cualitativo pone en evidencia el malestar docente debido a sobrecarga laboral, falta de reconocimiento y de tiempo de preparación de clases, bajos sueldos, entre otros. En este escenario, ha quedado del todo inexplorado de qué manera las políticas de rendición de cuenta, están afectando directa o indirectamente la vida presente y futura de los principales beneficiarios del sistema educativo y en qué tipo de experiencias y de aprendizajes se traducen en su pasar cotidiano. En otras palabras, los niños son los grandes ausentes del amplio debate académico y político sobre la educación en Chile.

Este vacío no es propio de los estudios sobre la realidad chilena solamente. Hay una notoria escasez también a nivel internacional de literatura que muestre la realidad de los niños como receptores de la cultura de la estandarización y de la performance. Sin 
embargo, destacan, en este sentido, los trabajos de Youdell (2004), Bialostok y Kamberelis (2010), Bradbury (2013) y Stahl (2015). Estos autores describen el tipo de identidad o de identidades que los niños construyen como consecuencia de organizaciones educativas y prácticas de enseñanza plasmadas por el neoliberalismo en educación.

En Chile, en cambio, la pregunta sobre la formación de la subjetividad de los aprendices en un contexto donde la calidad de la educación está profundamente marcado por políticas neoliberales, ha sido escasamente formulada. El trabajo de Carrasco (2014) muestra un claro intento de posicionarla en el debate al proponer que las políticas de privatización, accountability, estandarización y examinación intensiva sean vistas como una nueva forma de gubernamentalidad, que produce cierto tipo de sociedad y de sujetos y, en particular, refiriéndose a los estudiantes, "sujetos respondedores de pruebas, uniformizados y desprovistos de una experiencia educacional sustantiva" (Carrasco, 2014: 9).

Lo que se busca en este trabajo es ahondar en la pregunta por la formación del sujeto escolarizado, específicamente en un contexto de inefectividad educacional, sobre la base de una etnografía escolar en una escuela básica municipal de la Araucanía. Esta se realizó en el marco de un estudio comparativo de dos escuelas, una particular subvencionada en contexto rural mapuche y la otra municipal ubicada en un sector urbano de la Araucanía. El análisis se centró en las prácticas sociales que dan vida a la cotidianidad escolar en dos "comunidades de práctica" (Wenger, 1998) con el objetivo de comprender las diferencias en términos de aprendizaje desde una perspectiva no centrada en los resultados académicos, sino en las relaciones y en la identidad de los aprendices.

El supuesto que subyace a este enfoque es que en la vida escolar están en juego "formas de ser" que, inseparables de las "formas de hacer y conocer", son moldeadas en las actividades cotidianas. Estas, tal como plantean Herrenkohl y Mertl (2010), se construyen en la negociación ente individuos y el mundo social e incluyen intereses, motivaciones, compromisos emocionales y juicios sociales y personales sobre lo que es válido y tiene sentido aprender. En otras palabras, lo que los estudiantes aprenden en la educación formal no solamente está lejos de restringirse al ámbito académico o al de la socialización intencionada por los agentes educativos, sino además es el producto de una negociación, no privada de tensiones y conflictos, entre las expectativas de los educandos y las de los educadores.

Diversos estudios en el ámbito de la antropología y etnografía de la educación han puesto en evidencia los procesos y las dinámicas de la construcción de la persona en contextos educativos formales (Levinson et al., 1996), algunos mostrando de brillante manera las resistencias que manifiestan los estudiantes respecto al proyecto de socialización institucional al cual están sometidos (Evans, 2006; McLaren, 1986; Willis, 1977; Wolcott, 1974). Por otra parte, los estudios de microetnografía sobre las formas de participación en el aula han develado también el carácter intrínsecamente social de las interacciones rutinarias que se despliegan durante las actividades didácticas (Cazden et al., 1972; Erickson, 1996; McDermott, 1977; Mehan, 1979). En línea con esta tradición de estudios antropológicos de la educación, este trabajo pretende poner a luz las formas de participación de los niños de un VI año de básica en una escuela municipal de una ciudad de la Araucanía ${ }^{1}$. Busca indagar en las formas de ser y hacer que construyen los

Por razones de confidencialidad, en este trabajo se refiere a la escuela y a los profesores por medio de pseudónimos. También, por el compromiso de anonimato asumido con la escuela no se entrega información más específica sobre la ubicación del establecimiento. 
estudiantes durante su pasar por las largas horas de clase desde una perspectiva que mira a la participación en las situaciones de aula como el auténtico aprendizaje que se logra en el contexto escolar. Sin embargo, contrariamente a los estudios microetnográficos, esas situaciones son analizadas y reconstruidas desde una perspectiva holística que se sustenta en la etnografía escolar conducida durante el año escolar 2012. Es decir, se postula que el aprendizaje situado (Lave \& Wenger, 1991) de los niños se configura a partir de un escenario social y político y que este, en buena medida, plasma el tipo de interacciones y dinámicas de la escuela donde se centra este estudio.

\section{UN ESTUDIO DEL APRENDIZAJE EN LA COMUNIDAD DE PRÁCTICA ESCUELA}

El abordaje de la escuela como comunidad de práctica se basa en un interés por el aprendizaje como un fenómeno social. En particular, se centra en la idea que la práctica social es una dimensión intrínseca del aprendizaje y que, a su vez, el aprendizaje es un componente propio de la práctica social.

Por práctica social entendemos una actividad o un sistema de actividades sobre las cuales el grupo humano involucrado en ella/s tiene y construye significados compartidos. Esos significados pueden ser explícitos o implícitos, conscientes o inconscientes, pero en cualquier caso actúan como motor y dirección de la práctica facilitando su reproducción en contextos dados más allá de las divergencias o agencias individuales. La reproducción es una característica específica de la práctica, es decir, como plantea Bourdieu (1977), esta consiste en un conjunto de esquemas de percepción y acción que se repiten y se reactualizan en la acción misma de los sujetos.

Cuando decimos entonces que el aprendizaje es un componente de la práctica social, nos referimos a que el primero dice relación con el sentido que le vamos dando a las situaciones en las cuales estamos involucrados y a todos los elementos simbólicos y materiales que las conforman. Es la construcción y transformación de ese sentido que permite que la práctica se constituya como tal, esto es, dando lugar a corporalización de un conjunto de esquemas de percepción y acción. En otras palabras, tal como plantea una perspectiva fenomenológica del conocimiento y del aprendizaje (Ingold, 2001; Lave \& Wenger, 1991; Marchand, 2010; Toren, 2001, entre otros)for their interdependence is not trivial, but vital. The aim of this volume is to progress anthropologylu2019s thinking about human knowledge by exploring the interdependence of nurture with nature; and more specifically the interdependence of minds, bodies, and environments. This introductory essay begins with an overview of the (often conflicting, nuestros cambios en la comprensión del mundo no constituyen un momento a parte de nuestro actuar e interactuar en él, sino se va "haciendo" en la medida que estamos y actuamos en el medio. El medio es principalmente social, es decir, está hecho de las personas con las cuales interactuamos y de los lugares y artefactos que están cargados de significados socialmente compartidos, por lo que aprender es un proceso inherentemente "situado" en un espacio intersujetivo y consiste en una forma de "participar" (estar, ser y hacer) en él (Lave \& Wenger, 1991).

La escuela como comunidad de práctica es, entonces, un espacio intersubjetivo peculiar donde se construye el aprendizaje. Un aprendizaje que poco tiene que ver con lo que se diseña o se intenciona, sea en el currículum o en el proyecto educativo. Más bien 
se trata del aprendizaje que se genera en el día a día de las relaciones entre los actores de un espacio social que se dispone en torno a una "empresa común", como plantea Wenger (1998). Evidentemente, mucho de ese aprendizaje dice relación con lo que está estipulado justamente por esa empresa común, a través de los diferentes mecanismos de "reificación" como son el currículum, el proyecto educativo, las planificaciones, los procedimientos administrativos, entre otros. Estos mecanismos están diseñados para normalizar el funcionamiento de todas organizaciones escolares, generando en todas las escuelas municipales de Chile, por ejemplo, un setting y un modus operandi similar. Sin embargo, son las situaciones prácticas donde las ideas, procedimientos, normas y planes se materializan, y son estas las que, en última instancia, dan forma al aprendizaje.

Concretamente, lo que caracteriza una comunidad de práctica son los siguientes componentes: a) actividades que mantienen involucradas a un grupo de personas; b) significados compartidos sobre esas actividades; c) relaciones intersubjetivas regulares y estables. Estos componentes están claramente entrelazados y sobre ellos se construye tanto un corpus de conocimiento común que se renueva y transforma por medio de las relaciones intersubjetivas, como una identidad propia de los participantes de esa comunidad. Desde esta perspectiva, aprender en la escuela es involucrarse de manera paulatina en el sistema de relaciones, actividades y significados que caracterizan una comunidad de práctica particular y construir una forma de ser, en cuanto participante más o menos competente en esa comunidad.

De lo anterior sigue que la pregunta por el aprendizaje de los niños en la escuela requiere penetrar ese sistema de relaciones, actividades y significados por medio de un enfoque etnográfico, cuyos componentes centrales son la inmersión del investigador en la vida escolar por un tiempo prolongado y la "interpretación cultural" como fin último de la observación participante y de otras técnicas de recolección de datos cualitativos (Wolcott, 1985). Desde esta perspectiva -que entiende la etnografía no como un método sino como una forma de mirar e interrogar un contexto social- se llevó a cabo la investigación etnográfica en la escuela urbana Gregorio Urrutia. El trabajo de campo se realizó entre marzo y diciembre del 2012. Con un ritmo semanal, durante un día escolar completo (desde el inicio hasta el término de clases) quien escribe, investigadora responsable del estudio o en su reemplazo, la investigadora asistente del proyecto, participó de la vida del establecimiento por medio del seguimiento focalizado de un curso, el VI año. La observación se concentró, en buena medida, en las clases, aunque se participó también de los recreos, de las interacciones en la sala de profesores y en el pasillo y de las reuniones de profesores. Los datos provenientes de la observación fueron complementados y cruzados con el de las entrevistas en profundidad a niños y profesores. En su totalidad, el material etnográfico consta del registro escrito de 33 observaciones de clases de diferentes asignaturas -en su mayoría de lenguaje, matemática y ciencias sociales-; 25 entrevistas audiograbadas a niños y niñas de VI y 10 entrevistas a profesores y directivos, además de una entrevista grupal con los profesores de VI año.

El análisis de datos fue realizado de acuerdo a las siguientes dimensiones: prácticas docentes en el aula; formas de participación de los niños en la sala de clase; relación entre adultos y niños; experiencia y sentido de la escuela en profesores; experiencia y sentido de la escuela en los niños. Estas fueron examinadas y cruzadas con la finalidad de identificar las "complicidades implícitas" que constituyen el sustento de la comunidad de práctica escuela. Sin embargo, a partir de los resultados de la etnografía en la escuela 
Gregorio Urrutia no es posible identificar nítidamente una comunidad de práctica, sino más bien dos comunidades, la de los profesores por un lado y la de los estudiantes por otro, que se relacionan de manera antagónica y que se unen básicamente en función de un común sentido de sobrevivencia frente a una aparataje de disposiciones, procedimientos y estándares que para ambas resulta algo ajeno y a la vez enajenante. En la próxima sección entregaré algunos elementos de las dimensiones de análisis mencionadas junto con otros antecedentes generales de la escuela Gregorio Urrutia para, posteriormente, focalizarme en una de ellas, esto es las formas de participación de los niños en la sala de clase.

\section{LA ESCUELA MUNICIPAL GREGORIO URRUTIA}

En el año 2012, la escuela municipal Gregorio Urrutia cuenta con una matrícula de casi 300 alumnos que van desde el nivel prescolar hasta el VIII básico. Su población estudiantil es heterogénea, siendo el $30 \%$ de origen mapuche, el $34 \%$ proveniente de sectores rurales de la comuna y cerca del $60 \%$ del sector urbano. Más del $80 \%$ es considerada como "vulnerable". En el año 2011, la escuela fue catalogada como "emergente" debido a su bajo desempeño según las mediciones SIMCE y de acuerdo a la Ley 20.259 sobre el aseguramiento y fiscalización de la calidad de la educación. Lo anterior implicó la implementación de un plan de mejoramiento liderado por el equipo de gestión a cargo de la observación, evaluación y retroalimentación de las prácticas docentes en todos los cursos, pero por sobre todo del desarrollo del PAC (Programa de Apoyo Compartido) de I a IV año. Esta iniciativa implementada por el Ministerio de Educación a partir de 2011, apunta a entregar un conjunto de metodologías, estrategias y apoyo técnico para mejorar la efectividad de los procesos de planificación, enseñanza y evaluación. Concretamente, consiste en un sistema estandarizado que define de manera clara y estructurada contenidos, tiempos y estrategias docentes para cada unidad curricular. Su implementación en general, y en esta escuela en particular, no ha sido exenta de controversias por el grado de presión que ejerce sobre los docentes, pero también por su rígido formato que priva al profesor de la libertad de ajustar los procesos de enseñanza a necesidades y circunstancias particulares.

No se requiere un conocimiento muy acabado de la escuela Gregorio Urrutia para percibir que carece de un claro proyecto educativo. Pese a que el PEI plantee como misión la atención a la diversidad de los alumnos, la diversidad de origen de los niños es, de facto, ignorada, y el porcentaje de población mapuche presente resulta ser invisibilizado tanto en el ámbito curricular como en lo extra programático. Sin embargo, se ha consolidado un programa de integración escolar que cuenta con un equipo de profesores diferenciales y un programa de acompañamiento tanto en el aula de integración como en la sala para los niños diagnosticados con diversos tipos de trastornos o necesidades. Los profesores, interpelados sobre el proyecto educativo del colegio durante el focus group, tampoco manifiestan claridad ni consenso. Para algunos, el programa de integración daría el sello identitario al establecimiento, para otros este sería la certificación ambiental obtenida en 2008 y para otros, aun, el quehacer educativo simplemente estaría orientado a llevar a los estudiantes a la enseñanza media.

Las entrevistas individuales con los profesores dan cuenta de un bajo nivel de motivación hacia su trabajo docente. Los profesores muestran sufrir la presión de un sistema de rendición de cuentas que desprofesionaliza su labor. Ponen en duda su vocación 
por la enseñanza, que han perdido o nunca tenido, y se sienten abrumados por tener que cumplir con las expectativas del Ministerio de Educación, siendo que sus alumnos son, en su visión, vulnerables, no motivados a aprender y además carecen del apoyo de sus familias. Por lo demás, sienten que trabajan en un ambiente que no es especialmente agradable ni cooperativo, donde la innovación y la iniciativa de algunos es un problema para el resto al generar antecedentes para que todos se vean exigidos a mostrar un desempeño en áreas que excedan las tareas y funciones requeridas y evaluadas por el sistema de accountability. Se produce entonces un efecto de nivelación negativa (hacia abajo) funcional al "sentido de sobrevivencia" que orienta las prácticas docentes.

La alienación, el agobio y la falta de trascendencia en el quehacer docente se hacen evidentes en su desenvolvimiento en el aula. A partir de las múltiples observaciones de campo se configura una práctica de aula caracterizada por un formato estandarizado. El profesor o profesora básicamente cumple con todos los aspectos que se esperan y se promueven, a nivel oficial, en la práctica docente: se anota el objetivo en el pizarrón a principio de clase; se trabaja la materia, por lo general, a partir del texto; se estimula la participación de los niños con preguntas; se solicita periódicamente atención y orden a los niños de manera individual como colectiva; se instruye a los niños trabajar individualmente en el texto y se evalúan los conocimientos adquiridos con preguntas. En diversas ocasiones se registra la presencia de otro docente en el aula que apoya la labor del profesor que lleva la clase. Es el caso de la profesora diferencial, quien se sienta al lado de los niños que se considera tienen mayor dificultad, y del profesor de historia o de matemáticas quienes circulan por la sala ayudando a llamar la atención de los niños y explicando a los niños de manera individual. Pese a la presencia de dos profesores en la sala, resulta difícil mantener la atención de los 42 alumnos del VI año. Tal como se mostrará en el relato etnográfico, las clases, por lo general, se realizan de principio a fin sin mayores disrupciones, sin embargo, la mayoría de los estudiantes no lleva a cabo las actividades esperadas por los profesores y desarrolla formas propias de participación.

La relación entre profesores y estudiantes se caracteriza por un antagonismo que se expresa en la permanente tensión entre la afirmación de la autoridad del docente, por un lado, y la deslegitimación de su rol de parte de los alumnos, por otro. Esta última se materializa en gestos y actitudes explícitas de desafío, especialmente después de un reproche del docente, como una mueca a su espalda o una mirada de completa indiferencia. Al mismo tiempo, tal como se mostrará en la próxima sección, la creación de situaciones y actividades paralelas a la didáctica de parte de los estudiantes son de leerse como un claro intento, aunque menos consciente y directo, de subvertir la autoridad del docente y, conjuntamente, de desacreditar todo el sistema en que esta se sustenta.

Por su parte, los profesores están frecuentemente llamando la atención, pero la mayoría de las veces ignoran esas reacciones desafiantes así como esas situaciones paralelas que los niños crean durante la clase hasta que las últimas no se convierten en un claro impedimento para llevar a cabo su planificación didáctica. Cuando este es el caso, especialmente con un profesor, se han observado reacciones violentas como golpear la mesa con un palo para recuperar el control de la clase. Esta situación de abierto conflicto se explicita también en las ocasiones en que algunas profesoras piden una suerte de tregua: cuando los estudiantes están especialmente desordenados o hacen dificultosa la realización de la clase, algunas profesoras ya no invocan su rol de autoridad, sino apelan a la relación humana y al sentido de piedad de los estudiantes, suplicándoles: "Silencio, por favor, chicos, que hoy me siento mal". 
En ocasiones es posible observar una relación humana y afectiva entre algunos profesores y algunos estudiantes. Esta, eventualmente, puede ser interpretada como una forma para soslayar la desigualdad de poder entre ambas partes y para alivianar la tensión que genera el antagonismo que se produce durante las clases. El vínculo afectivo con los docentes es reportado en las entrevistas como uno de los aspectos que los estudiantes aprecian de su pasar en la escuela, la que se valora, por sobre todo, como espacio de socialización. Sin embargo, en las entrevistas los niños dan cuenta de un nivel de motivación muy bajo hacia la escuela y, muy notoriamente, expresan una visión crítica hacia la forma en que se les enseña y hace clase. Muchos de ellos declaran no amar algunos ramos que son las asignaturas en que "no le explican bien" o "le explican a los más inteligentes no más". Esta percepción refuerza lo que emerge de las observaciones de clase donde los niños parecen no tener acceso a los contenidos que trabaja el profesor y, por lo mismo, frustrarse. Esa misma frustración es la que los llevaría a "inventar" cómo llenar el tiempo en el aula.

\section{FRUSTRACIÓN, SOBREVIVENCIA Y RESISTENCIA EN LA SALA DE CLASE}

Llego justo a tiempo para el inicio de la clase de matemática. Como siempre busco un asiento disponible entre los bancos del fondo de la sala y encuentro uno delante de unas niñas que están haciendo pulseras con hilos de lanas de colores. Siguen así cuando empieza la clase oficialmente. Pregunto por qué hay otro profesor junto con la profesora María, docente de matemática. Las niñas me explican que a los que les cuesta más la clase se van con el profesor ayudante, mientras los más inteligentes se quedan aquí con la profesora. La profesora escribe los objetivos de la clase en la pizarra:

1. Validar la solución de una ecuación de primer grado.

2. Resolver problemas por medio de ecuaciones.

La profesora trata de llamar la atención preguntando qué es una ecuación, las respuestas se demoran en llegar, son pocas y parecen improvisadas. La profesora pregunta más de una vez pero se mantiene una cierta distracción, quienes no están "en otras", como conversando o haciendo pulseras, se concentran en anotar el objetivo en sus cuadernos. La profesora les pide que primero pongan atención y después escriban.

La profesora anota una ecuación y hace preguntas constantemente, el otro profesor está de pie atrás e interviene con breves comentarios, solicita atención. Las chicas atrás -veo- que siguen tejiendo pulseras. De a poco el murmullo se calma y algunos niños enganchan con las preguntas de la profe e intentan responder. La profesora les recuerda varias veces que tienen que "indicar", o sea levantar la mano, en vez de llegar e intervenir.

La profesora elogia las respuestas de quienes parecen haber entendido cómo resolver la ecuación, "muy bien" dice. La chica a mi lado le pregunta a la que está adelante ¿tú entendiste? Y ella pone cara de interrogación y complicación. La profesora anota más ecuaciones para que los chicos las resuelvan por su cuenta. Otros minutos de murmullo en que varios se desenganchan nuevamente, conversando con el compañero del lado o de la fila de atrás o de adelante.

Algunos chicos sí se ven concentrados en la tarea con el foco en su cuaderno, la mayoría alterna momentos de concentración con largos momentos en que le comentan algo al compañero, golpean rítmicamente el lápiz sobre la mesa o cuaderno, chupan un lolipop o le tiran una pelotita de papel al compañero. 
La profesora hace nuevos llamados de atención, pero sigue el murmullo. Se dirige hacia un grupo que hace más desorden y boche jugueteando a golpear los lápices sobre la mesa. Pero ella se fija en uno de ellos, que no es el que hace más ruido, sino un chico que ha estado casi siempre dirigido hacia sus compañeros y no trabaja en el cuaderno. De hecho ella lo reprocha por no tener cuaderno. “¿No te importa nada?”, le pregunta. También llama la atención a dos alumnos que no tienen nada anotado en su cuaderno y sube el tono antes de volver a ubicarse cerca del pizarrón. La chica a mi lado vuelve a preguntarle a la de adelante si ahora entendió, porque ella no, dice. Me sorprendo a mí misma con una pisca de angustia. Me parece revivir lo que solía sentir cuando yo también, lenta para los números, quedaba colgada en las clases de matemática.

Muy pocos ponen atención a la nueva demostración en el pizarrón. Llega un momento en que nadie está mirando y la profe se acerca más al chico en el pizarrón y despacio monitorea y apoya lo que está haciendo. Después insta al chico a explicar lo que hizo. El chico espera sugerencias de los compañeros para proceder ¿Por qué esperas que te soplen? La profesora vuelve a explicar cómo resolver las ecuaciones: "Se juntan los números sin x para un lado y las x para el otro (...)". Las demostraciones en la pizarra tienen poco de demostración, son más bien ejercicios individuales hechos en un lugar público, donde el resto si quiere puede ver. Ahora no hay casi nadie que esté siguiendo lo que el chico hace en el pizarrón. La chica a mi lado dice que le cuesta entender, le sugiero preguntarle al profe pero no se atreve. La profe apoya al "demostrante" mientras la clase se ha convertido en un buen caos, pese a que el profesor de "de refuerzo" circula por los puestos.

La profesora llama nuevamente la atención al curso donde se ha levantado nuevamente mucho murmullo y confusión: ¿Cuántos están poniendo atención? Cuatro o cinco y nada más!’”2

La profesora María es una docente muy respetada por sus colegas. Lleva una larga trayectoria como docente, de hecho este es su último año de ejercicio en la profesión antes de jubilar. Enseña en la escuela hace cerca de 15 años. El Director me habló de ella antes de que la conociera, presentándomela como una excelente profesora. La primera impresión al observarla es de una docente muy seria y con una consolidada metodología de enseñanza de las matemáticas. Su trato con los alumnos es, normalmente, distante y formal y muy abocado a la labor académica. Con cierto éxito (en comparación con otros docentes) consigue contener la tendencia al desorden de los alumnos y sus diversas formas de resistencia a la actividad didáctica. Los estudiantes, por ejemplo, no suelen pararse de sus puestos para conversar con los compañeros como puede ocurrir en otras clases. Sin embargo, pese al relativo respeto del cual goza, no es querida por los niños. Según su percepción, esta profesora haría clases siguiendo el ritmo de aprendizaje de algunos pocos en la sala, sin importarle que otros y otras no puedan seguirlo. Por lo mismo el ramo de matemática termina siendo, para los niños, "el que menos les gusta".

Los niños expresan de manera inequívoca lo que aparece evidente también a ojos de un observador, como la etnógrafa, que se ubica en las filas de atrás de la sala de clases, lejos de los alumnos modelos que escogen los primeros puestos. Estos son los que gozan del privilegio de tener una natural inclinación por los números y captan con facilidad, e incluso entusiasmo, los desafíos planteados. La gran mayoría de los niños muestra una clara conciencia de la frustración que les genera una práctica pedagógica que está dirigida a unos pocos, a los que entienden a la primera explicación y que serían "los más inteligentes". Por lo mismo, dicen aburrirse y pasarlo peor que en otras clases.

Este relato etnográfico es un compilado de las notas tomadas durante las clases de matemática en las siguientes fechas: 17 de agosto 2012; 30 de agosto 2012; 6 de Septiembre 2012. 
Ahora, en base a lo observado en las otras clases (Lenguaje y comunicación, Historia y geografía, Inglés, Arte y Tecnología), puede concluirse que la frustración ante la falta de comprensión de la materia y del "sentido" de esta es algo generalizado. Raramente los estudiantes muestran genuino interés y entusiasmo por los contenidos tratados, excepto los pocos que permiten que la profesora tenga un mínimo de interlocución y de diálogo para poder realizar su clase. Pero lo que son bastante más notorias en otras clases son las maneras propias de los niños de invertir el tiempo didáctico en actividades alternativas a la que es liderada por la profesora. Una de ellas es la circulación y consumo de membrillos con sal. Un chico, aparentemente ya reprochado una vez por haber traído membrillos, los saca de una bolsa al empezar la clase de inglés y los reparte a quienes le van solicitando junto con una bolsita de sal, por lo que membrillos y sal van pasando de mano en mano, con un murmullo contenido en la clandestinidad, mientras la profesora realiza su clase, aparentemente sin darse cuenta de nada. Otra situación llamativa es la de un niño que, al empezar la clase de historia, da inicio a la larga tarea de picar en pequeños trozos su goma de borrar de la clásica marca alemana que se caracteriza por su firmeza, lo que continúa de manera metódica durante toda la hora.

Pareciera que los niños vienen "equipados" para afrontar las largas y aburridas horas de clase y cuentan con un amplio abanico de estrategias para sobrellevarlas. En este sentido, tejer pulseras de lana, comer membrillos con sal, destruir metódicamente una goma de borrar; y también acordar las posiciones en el próximo partido de futbol, hacer una tarea para otra clase o simplemente conversar de cualquier cosa tratando de no ser identificado y/o censurado por el docente, pueden ser acciones interpretadas como expresiones de una respuesta creativa y resiliente a la frustración y al tedio que experimentan durante las horas de clase. Aquí podría encontrarse también la clave para comprender el exacerbado criticismo de los estudiantes hacia la profesora de matemática. La docente, al tener más autoridad que otros por su trato serio y formal, no permite una "apropiación" del espacio de clases por parte de los estudiantes. Las medidas para sobrevivir al tedio y esquivar la frustración no pueden ser implementadas en sus clases, por lo que esas sensaciones son vividas en su integridad, sin ningún mecanismo de filtro o mitigación.

El desenvolvimiento de los talleres del jueves en la tarde es una clara muestra de cómo el evitar "someterse" en pleno a la actividad didáctica para establecer formas propias de participación se ha convertido en una práctica establecida de los estudiantes. Estos se inscriben en uno de ellos, pudiendo elegir entre una variedad de actividades pensadas para ofrecer un espacio grato de desarrollo de habilidades no vinculado a las asignaturas: manualidades, francés, medioambiente, matemática entretenida, cine, entre otros. Con la excepción de algunos talleres, el desencanto y la falta de involucramiento de los estudiantes predominan en estas instancias, junto con los intentos de substraerse a la actividad programada. Es decir, se reproducen las mismas dinámicas que en las clases, pero con la atenuante de que, tratándose de horas no lectivas, la tensión en torno al control de la participación es mínima y el antagonismo profesor-estudiante aparece diluido. Los estudiantes aprovechan plenamente el mayor grado de libertad del cual gozan para imponer, con la seguridad de no sufrir ningún tipo de consecuencia, sus propios intereses contra los que, supuestamente, habrían sido institucionalizados por medio de su elección de los talleres. Lo anterior es particularmente evidente en el taller de cine, donde se proyectan principalmente comedias de amplia popularidad, pero que, sorprendentemente, no captan la atención de los estudiantes. Estos, en su gran mayoría, prefieren conversar, jugar "al colgado", mirar videos en el celular y llenar de preguntas a la etnógrafa. 
Reproduciendo un patrón propio de la niñez, los estudiantes buscan (y finalmente encuentran), el componente lúdico presente en el hecho de romper o evadir las reglas puestas por los adultos. Sin embargo, lo peculiar aquí es que esta tendencia o hábito se aplica también a esos casos donde las formas de participación requeridas o normadas por los adultos están diseñadas para favorecer directamente los intereses de los niños. Lo que ocurre durante el taller de cine ofrece una clara demonstración de esta práctica.

Otra evidencia es la resistencia a salir de la sala a la hora del recreo. Es decir, mientras los estudiantes se las ingenian durante toda la hora de clase para encontrar la manera de salir de la sala, en la hora del recreo, cuando se les solicita salir para descansar, jugar y distraerse, algunos ponen en acto diferentes tácticas para quedarse adentro en el momento en que la auxiliar viene a cerrar la sala con llave. Con cierto orgullo me cuentan los mismos detractores que suelen esconderse en el closet o acurrucarse debajo de las mesas para no ser vistos. Me dicen que no les gusta ir para afuera porque hace mucho frío. En realidad, los alumnos no tienen la obligación de salir al patio porque durante el recreo, muchos lugares al interior del establecimiento son accesibles a los niños (biblioteca, pasillos, salas de juego, entre otros). Sin embargo, prefieren quedarse en la sala haciendo pulseras de hilos de lana, por ejemplo, actividad que, tal como se ha mostrado, no es privativa de los momentos de esparcimiento, sino se lleva a cabo durante las horas de clase también. Estos antecedentes confirman la tendencia generalizada a esquivar las normas con una motivación que trasciende la mera sobrevivencia a la frustración y tedio de las horas lectivas. Una posible explicación es que los estudiantes hayan internalizado una "falta de sentido" en las normas y pautas escolares. Es decir, asumen que, por lo general, toda la participación de la vida escolar no está hecha para complacer a los niños. Esto los llevaría a desconfiar de aquellas prácticas que son promovidas, al contrario, como placenteras o auténticamente atractivas para ellos. Prefieren aplicar el principio de la resistencia y seguir buscando el sentido que normalmente no encuentran en las situaciones rutinarias institucionalmente creadas en el mismo hecho de subvertirlas.

\section{CONSTRUYENDO LA “IDENTIDAD DEL EXCLUIDO” EN LA SALA DE CLASE}

Dado el contexto anteriormente descrito, cobra relevancia la pregunta por el aprendizaje de los niños. No hay dudas de que su mayor ocupación y "pre-ocupación" durante las horas de clase, que son las que llenan la mayor parte del día escolar, no está en las actividades académicas. Es decir, aun cuando físicamente están realizando las tareas solicitadas lo hacen de manera mecánica, sin entender o apreciar lo que están haciendo. Por lo mismo, parecen buscar ansiosamente la manera de evadir, aunque sea por unos fugaces minutos, las actividades académicas e involucrarse en otras que sean de algún modo significativas para ellos. Mi hipótesis es que el grado de significatividad o sentido de las otras formas de participación en clase no necesariamente estaría relacionado con el contenido mismo de esas actividades alternativas, sino con el mero hecho de realizarlas, es decir con el acto de liberación y resistencia que representa el no cumplir con lo solicitado por la institución y crear microespacios de participación hechos a la propia medida. Con la motivación implícita de ser protagonistas en el diseño e implementación de "espacios de sentido propio" y contrapuestos al institucional, los niños ponen en acto formas de participación que se convierten en aprendizajes específicos. 
Aprenden, en primer lugar, a desenmarcarse de la estructura de roles, funciones, actividades en las cuales están insertos. Más aún, casi de manera condicionada, aprenden a desconfiar de la agenda institucional, en la creencia de que nada auténticamente bueno e interesante esta puede ofrecerles, aun cuando constantemente el aparataje institucional trata de venderles lo contrario. Las ideas meritocráticas de que la educación escolar es el trampolín para el éxito en la vida y que todo se puede lograr mediante el esfuerzo individual son el pilar sobre el cual se fundamenta la educación pública y son reiteradas constantemente en las prácticas discursivas y no discursivas de los adultos en el colegio. Mostrar a los estudiantes un cuaderno limpio y ordenado de una alumna y declarar que la niña tiene el éxito asegurado, como ocurrió en una ocasión, es solo un ejemplo de estas prácticas. Sin embargo, la misma institución que pone toda la apuesta por el progreso social en los niños y en sus capacidades individuales, no genera las condiciones para que estas realmente puedan desarrollarse. Al contrario, mediante prácticas de aula excluyentes la escuela produce sentimientos de frustración y promueve mecanismos de evasión. En este escenario, la desconfianza de parte de los niños hacia la institucionalidad y la deslegitimación de la autoridad de sus más próximos representantes, los docentes, parecen ser reacciones esperables. Pero los niños no aprenden solo a desconfiar de la institucionalidad, aprenden también, impulsados por la frustración y la desconfianza, a "arreglárselas", es decir a utilizar una instancia oficialmente diseñada con ciertos fines, para otras actividades y otros fines de su interés. Lo anterior implica también la habilidad, que se expresa en la puesta en acto de diferentes estrategias, para evadir o romper las normas.

Por otra parte, tal como se ha planteado anteriormente, el aprendizaje involucra no solo formas de hacer sino también de ser (Herrenkhol \& Mertl, 2010), o como plantean Lave y Wenger (1991) una identidad. El siguiente extracto del texto que ya se ha convertido en un clásico de la literatura sobre aprendizaje desde una perspectiva social y fenomenológica, explica claramente este punto:

\begin{abstract}
Siendo un aspecto de la práctica social, aprender involucra la persona integralmente; esto implica no solo una relación con actividades específicas, sino también una relación con comunidades, implica convertirse en un pleno participante, un miembro, un tipo de persona. En esta óptica, aprender solo parcialmente $-\mathrm{y}$ frecuentemente de manera accidental- implica adquirir la capacidad de involucrarse en nuevas actividades, desempeñarse en nuevas tareas y funciones, dominar nuevas comprensiones. Actividades, tareas, funciones y comprensiones no existen de manera aislada; estos son parte de un amplio sistema de relaciones en el cual tienen significado. Estos sistemas de relaciones emergen de y a la vez son reproducidos y desarrollados al interior de comunidades que son, en parte, sistemas de relaciones entre personas. La persona está definida y, a su vez, define esas relaciones. Por lo tanto, aprender implica convertirse en una persona diferente en relación a las posibilidades que permiten estos sistemas de relaciones. Ignorar este aspecto del aprendizaje es pasar por alto el hecho que aprender involucra la construcción de identidades (Lave y Wenger, 1991: 53) ${ }^{3}$
\end{abstract}

Desde esta perspectiva, postulo que los niños en la escuela estudiada, no solo quedan excluidos de los conocimientos curriculares que se espera que manejen como resultado de su frecuentación de la escuela, y como consecuencia se autoexcluyen de las formas de participación promovidas por la institución. Además, desarrollan una identidad del excluido que se revela también en episodios en que "tiran la esponja" frente a las tareas a desarrollar,

Traducción propia. 
o muestran casi satisfacción por sus malas notas y mal comportamiento. "Puse puras cosas tontas, no se la voy a pasar, porque no entendí, no estudié nada", ${ }^{4}$ contesta con displicencia un estudiante a la etnógrafa que le pregunta cómo le fue la prueba. Esta estudiante pareciera haber internalizado su condición de excluida y haber hecho de ella un nicho identitario en el cual, posiblemente, ya no se siente tan incómoda. No tiene mayor implicancia no entregar la prueba, pues esto no cambiaría su situación. Con su respuesta ella invalida el sistema que la excluye y la hace sentir excluida y, conjuntamente, desacredita su posición de excluida en el mismo. En otras palabras, la estudiante asume y la vez consolida su condición de excluida, pero al mismo tiempo, con su indiferencia, le resta valor y relevancia.

En este marco surge la pregunta respecto a si es posible pensar que las formas en que aprenden a relacionarse con la institucionalidad escolar se trasladen a su interacción con la institucionalidad pública en general (Estado). En otras palabras, ¿cómo se proyecta en la vida ciudadana de los estudiantes la identidad del excluido plasmada en una experiencia de escolarización como la que reporta esta investigación?

En Chile, la participación de los estudiantes en el aula y en la escuela como un componente central de la formación ciudadana de los niños y jóvenes ha sido ya objeto de atención, tanto en la literatura como en las políticas educacionales. El cambio de paradigma desde la educación cívica a la formación ciudadana con su nuevo énfasis en el "desarrollo de competencias (conocimientos, habilidades y actitudes) para participar cívica y políticamente" (Cox et al., 2005: 6), ha implicado igualmente mirar no solo a los contenidos abordados, sino también al clima escolar y de aula, es decir, a la calidad de la convivencia entre los estudiantes, las prácticas pedagógicas, las oportunidades para la participación en la sala de clase, entre otros (Cerda et al., 2004; Bonhomme et al., 2015; Martinez \& Cumsille, 2015; Ministerio de Educación de Chile [MINEDUC], 2005; Reyes et al., 2013). Lo anterior se ha traducido, por ejemplo, en la incorporación en el currículum de Historia, geografía y ciencias sociales de la expresa indicación de promover la participación ciudadana con "un aprendizaje por medio de la acción" que implique "acciones cotidianas al interior de la escuela y el hogar" (MINEDUC, 2012a: 181). Por otra parte, las Bases Curriculares de Orientación apuntan al desarrollo de capacidades y actitudes para la convivencia, la participación y la pertenencia por medio de las mismas instancias escolares como el Consejo de Curso (MINEDUC, 2012b).

Sin embargo, desde una perspectiva que concibe el aprendizaje como una forma de participación en una comunidad de práctica, el clima y la convivencia son, más que un factor influyente, la médula del desarrollo de habilidades y construcción de conocimiento. Esta visión es consistente con la idea de "ciudadanía como práctica" impulsada por Gert Biesta y Robert Lawy (Biesta et al., 2009; Lawy, 2014; Lawy \& Biesta, 2006) según los cuales la ciudadanía no debe ser entendida como el resultado de un proceso de adquisición de conocimientos, ni como un estatus que los niños se preparan a alcanzar en su vida adulta. Esta concepción es excluyente porque priva a los niños y jóvenes de su condición actual de ciudadanos y pone el énfasis en las estrategias o intervenciones a implementar para convertirlos en miembros de la sociedad civil y no en las formas en que ellos en su vida diaria ejercen y experimentan su pertenencia a los diferentes contextos (escuela, familia, grupos de pares) en que participan.

Notas de campo, 10 de agosto 2012. 
La idea de ciudadanía como práctica sugiere que la ciudadanía se pone en juego en las situaciones cotidianas en que los niños y jóvenes ejercen su agencia dentro de los sistemas de relaciones de los cuales son parte. En particular, se configura a partir de la intersección entre "contextos", "relaciones" y "disposiciones" que dan lugar a las diferentes trayectorias de vida de las personas (Biesta et al., 2009). Desde un enfoque que se inspira en el pensamiento de Dewey sobre la centralidad de la experiencia personal tanto para el aprendizaje como para el ejercicio de la democracia, Biesta et al. proponen mirar a las trayectorias de vida de los jóvenes como formas en que se despliega y a la vez se construye su condición de ciudadanos, para concluir lo siguiente:

Para los hacedores de políticas, uno de los más importantes mensajes a extraer del enfoque delineado en este artículo es que todo intento de mejorar la ciudadanía de los jóvenes debiera empezar por el mejoramiento de la calidad de la democracia de las comunidades y prácticas que hacen las vidas de las personas. Después de todo, es la calidad de la actual condición de la ciudadanía de las personas jóvenes -e.g. las oportunidades para participar y ponerse en juegoque es de crucial importancia para hacer, ser y aprender en pos de la democracia" (Biesta et al., 2009: 15$)^{5}$

Desde esta perspectiva, que vincula la participación de los niños a su vida escolar con su formación como ciudadanos, es que propongo leer las formas descritas de resistencia de los niños en la sala de clase de la escuela Gregorio Urrutia. En otras palabras, parece legítimo suponer que la identidad del excluido que muchos niños van formando durante el transcurso de sus vivencias en el aula sea no solo el producto de la exclusión generada por prácticas pedagógicas inadecuadas, sino que también configure un conjunto de disposiciones y significados que conduzcan a la autoexclusión de la vida democrática y de la participación civil. Quiero resaltar que, si bien la formación a la ciudadanía no es de restringirse a la escuela, sino que atañe todas las instancias de la vida social de un niño, tal como plantean Lawy y Biesta (2006), la escuela tiene un papel preponderante no tanto por su misión educativa como por la función inclusiva que se le adscribe: por medio de la educación pública todos los niños tendrían iguales oportunidades de integrarse satisfactoriamente en el mundo laboral y en la vida social. Las diferencias en el grado de éxito deben atribuirse, con alguna atenuante, a las características y/o al mérito de los propios estudiantes o de sus familias. En otras palabras, pese al sistema de rendición de cuenta, los discursos y prácticas que se reproducen en la mayoría de las escuelas muestran como la responsabilidad del éxito o del fracaso escolar de los niños y jóvenes es considerada, sustancialmente, como algo ajeno a la gestión institucional y al quehacer de los profesores.

Esta concepción, basada en convicción o conveniencia, se vincula a la noción de escuela como universal dispensadora de herramientas para el logro individual y el ascenso social. Paradójicamente, las prácticas excluyentes de los docentes de la escuela Gregorio Urrutia parecen tener este supuesto detrás: los profesores cumplen con realizar la didáctica esperada según los formatos estandarizados por el Ministerio y fiscalizados por el sistema de rendición de cuentas; está en los estudiantes incorporarse a las actividades y, con su participación, hacer que la clase tenga los logros esperados. Por otra parte, las dificultades de algunos son asunto de los encargados de ejecutar el Programa de Integración, y excepcionalmente se considera que deban ser abordadas mediante una adecuación de las

Traducción propia. 
prácticas docentes. El resultado de este esquema es, tal como se ha mostrado con esta etnografía, la exclusión y autoexclusión de los niños del aprendizaje y de la participación de los niños y el desarrollo de nuevas formas de participación que se construyen en resistencia a las institucionales. Los niños resultan excluidos justamente de la instancia que estaría diseñada para incluirlos en la sociedad. Considero que esa paradoja es especialmente nefasta para la formación ciudadana de los niños, ya que afecta su sentido de pertenencia a un colectivo y su confianza en las instituciones y en las normas de convivencia que estas establecen, llevándolos a buscar y a preferir formas "no legítimas" de participar en la comunidad cívica. En conclusión, sugiero que la atención al punto de vista de los niños, a su experiencia y participación en las aulas escolares es un paso ineludible, no solo para mejorar la calidad de la educación chilena sino también para hacer frente a los bajos índices de expectativas de participación política y a la actual crisis de confianza en la institucionalidad vigente.

\section{REFERENCIAS BIBIOGRÁFICAS}

Acuña, F., Assaél, J., Contreras, P., \& Peralta, B. (2014). La traducción de los discursos de la política educativa en la cotidianeidad de dos escuelas municipales chilenas: La metáfora médica como vía de análisis. Psicoperspectivas. Individuo y Sociedad, 13(1), 46-55. doi:10.5027/ psicoperspectivas-Vol13-Issue1-fulltext-363

Assaél, J., Cornejo, R., Gonzalez, J., Redondo, J., Sanchez, R., \& Sobarzo, M. (2011). La empresa educativa Chilena. Educação \& Sociedade, 32(115), 305-322. doi:10.1590/S010173302011000200004

Astudillo, G., \& Imbarack, P. (2013). El sentido del mejoramiento escolar de los docentes en el contexto de políticas de apoyo externo. Pensamiento Educativo, 50(2), 132-146. doi:10.7764/ PEL.50.2.2013.18

Ball, S. (2003). The teacher's soul and the terrors of performativity. Journal of Educational Policy, 18(2), 215-228. doi:10.1080/0268093022000043065

Ball, S. J. (1998). Big Policies/Small World: An introduction to international perspectives in education policy. Comparative Education, 34(2), 119-130. doi:10.1080/03050069828225

Bellei, C. (2007). Expansión de la educación privada y mejoramiento de la educación en Chile. Evaluación a partir de la evidencia. Expansion of private schools and educational. Revista Pensamiento Educativo, 40(1), 285-311. Recuperado desde http://www.opech.cl/bibliografico/doc_ movest/BELLEI_Privado_vs_Publico_Pensamiento_Educativo.pdf

Bialostok, S., \& Kamberelis, G. (2010). New capitalism, risk, and subjectification in an early childhood classroom. Contemporary Issues in Early Childhood, 11(3), 299-312. doi:10.2304/ ciec.2010.11.3.299

Biesta, G., Lawy, R., \& Kelly, N. (2009). Understanding young people's citizenship learning in everyday life: The role of contexts, relationships and dispositions. Education, Citizenship and Social Justice, 4(1), 5-24. doi:10.1177/1746197908099374

Bonhomme, M., Cox, C., Tham, M., \& Lira, R. (2015). La educación ciudadana escolar de Chile 'en acto': prácticas docentes y prácticas docentes y expectativas de participación política de estudiantes. En C. Cox y J. C. Castillo (Eds.), Aprendizaje de la Ciudadanía. Contextos, experiencias y resultados (pp. 377-425). Santiago de Chile: Ediciones Universidad Católica de Chile.

Bradbury, A. (2013). Education policy and the "ideal learner": producing recognisable learnersubjects through early years assessment. British Journal of Sociology of Education, 34(1), 1-19. doi $: 10.1080 / 01425692.2012 .692049$

Carrasco, A. (2014). Mecanismos performativos de la institucionalidad educativa en Chile: 
Estudios Pedagógicos XLI, N Especial: 97-113, 2015

CONSTRUYENDO "LA IDENTIDAD DEL EXCLUIDO": ETNOGRAFÍA DEL APRENDIZAJE SITUADO DE LOS NIÑOS

EN UNA ESCUELA BÁSICA MUNICIPAL DE CHILE

pasos hacia un nuevo sujeto cultural. Observatorio Cultural, 15, 4-10. Recuperado desde http:// www.cultura.gob.cl/observatorio15/observatorio_cultural_n15.pdf

Carrasco, A., \& Martín, E. S. (2012). Voucher system and school effectiveness: Reassessing school performance difference and parental choice decision-making. Estudios de Economía, 39(2), 123-141. Recuperado desde http://www.redalyc.org/articulo.oa?id=22125058002

Cazden, C., John, V., \& Hymes, D. (1972). Functions of the Language in the Classroom. New York: Teachers College.

Cerda, A. M., Egaña, M. L., Magendzo, A., Santa Cruz, E., \& Vargas, R. (2004). El complejo camino de la formación ciudadana. Una mirada a las prácticas docentes. Santiago de Chile: LOM.

Corvalán, J. (2013). La narrativa educacional chilena y su proceso de transformación reciente. Folios, 37, 63-81.

Cox, C., Jaramillo, R., \& Reimers, F. (2005). Educar para la ciudadanía y la democracia en las Américas: Una agenda para la acción. New York y Washington: Banco Interamericano de Desarrollo. Recuperado desde http://www.educadem.oas.org/documentos/getdocument.pdf

Erickson, F. (1996). Going for the zone: the social and cognitive ecology of teacher-student interaction in classroom conversations. In D. Hicks (Ed.), Discourse, Learning and schooling (pp. 29-62). Cambridge, UK: Cambridge University Press.

Evans, G. (2006). Educational failure and working class white children in Britain. Basingstoke, Uk: Palgrave M.

Falabella, A. (2014). The Performing School: The Effects of Market \& Accountability Policies. Education Policy Analysis Archives, 22(70), 1-25.

Falabella, A., \& Opazo, C. (2014). Sistema de Aseguramiento de la Calidad y procesos de mejoramiento: una mirada desde la gestión educativa. Informe Ejecutivo. Santiago de Chile: Universidad Alberto Hurtado.

Herrenkohl, L., \& Mertl, V. (2010). How Students Come to Be, Know and Do. A Case for a Broad View of Learning. Cambridge and New York: Cambridge University Press.

Ingold, T. (2001). From the Transmission of Representations to the Education of Attention. In H. Whitehouse (Ed.), The Debated Mind. Evolutionary Psychology versus Ethnography. Oxford: Berg.

Lave, J., \& Wenger, E. (1991). Situated Learning: Legitimate Peripheral Participation. New York: Cambridge University Press.

Lawy, R. (2014). Education beyond socialization: on becoming and being a citizen-subject in everyday life. Discourse Studies in the Cultural Politics of Education, 35(4), 599-610. doi:10.1080/ 01596306.2013.871227

Lawy, R., \& Biesta, G. (2006). Citizenship-as-Practice: Educational Implications of an Inclusive and Relational Understanding of Citizenship. British Journal of Educational Studies, 54(1), 34-50.

Levinson, B., Foley, D., \& Holland, D. (Eds.). (1996). The Cultural Production of the Educated Person: Critical Ethnographies of Schooling. Albany: State University.

Marchand, T. H. J. (2010). Making knowledge: explorations of the indissoluble relation between minds, bodies, and environment. Journal of the Royal Anthropological Institute, S1-S21. doi:10.1111/j.1467-9655.2010.01607.x

Martinez, M. L., \& Cumsille, P. (2015). La escuela como contexto de socialización política: influencias colectivas e individuales. En C. Cox y J. C. Castillo (Eds.), Aprendizaje de la Ciudadanía. Contextos, experiencias y resultados (pp. 429-457). Santiago de Chile: Ediciones Universidad Católica de Chile.

McDermott, R. (1977). Social Relations as Contexts for Learning in School. Harvard Educational Review, 47(2), 198-213.

McLaren, P. (1986). Schooling as a ritual performance. Towards a political economy of educational symbols. London, Boston and Henley: Routledge.

Mehan, H. (1979). Learning Lessons. Social Organization in the Classroom. Cambridge and London: Harvard University. 
Ministerio de Educación de Chile. (2005). Informe Comisión Formación Ciudadana. Santiago de Chile.

Ministerio de Educación de Chile (2012a). Bases Curriculares. Geografía y Ciencias Sociales. Santiago de Chile. Recuperado desde http://curriculumenlinea.mineduc.cl/sphider/search. php?query\&t_busca $=1 \&$ results\&search $=1 \&$ dis $=0 \&$ category $=1$

Ministerio de Educación de Chile (2012b). Bases Curriculares. Orientación. Santiago de Chile. Recuperado desde http://curriculumenlinea.mineduc.cl/sphider/search.php?query\&t_ busca $=1 \&$ results \&search $=1 \&$ dis $=0 \&$ category $=1$

Niesche, R. (2015). Governmentality and My School: School Principals in Societies of Control. Educational Philosophy and Theory, 47(2), 133-145. doi:10.1080/00131857.2013.793925

Reyes, L., Campos, J., Osadon, L., \& Muñoz, C. (2013). El profesorado y su rol en la formación de los nuevos ciudadanos: desfases entre las comprensiones, las actuaciones y las expectativas. Revista Estudios Pedagógicos, 34(1), 217-237. doi:10.4067/S0718-07052013000100013

Stahl, G. (2015). Identity, Neoliberalism and Aspiration. Educating white working class boys. New York: Routledge.

Toren, C. (2001). The child in mind. In H. Whitehouse (Ed.), The Debated Mind. Evolutionary Psychology versus Ethnography. Oxford: Berg.

Valenzuela, J. P., Bellei, C., \& De los Rios, D. (2010). Segregación escolar en Chile. En S. Martinic y G. Elaqua (Eds.), ¿Fin de ciclo? Cambios en la gobernanza del sistema educativo (pp. 209-229.). Santiago de Chile: Orealc-Unesco. Pontificia Universidad Católica de Chile.

Valenzuela, J. P., Villarroel, G., \& Villalobos, C. (2013). Ley de Subvención Escolar Preferencial (SEP): algunos resultados preliminares de su implementación. Pensamiento Educativo: Revista de Investigación Educacional Latinoamericana, 50(2), 113-131. doi:10.7764/PEL.50.2.2013.17

Wenger, E. (1998). Communities of practice: Learning, meaning, and identity. New York: Cambridge University Press.

Willis, P. (1977). Learning to Labour. How working class kids get working class jobs. New York: Columbia University Press.

Wolcott, H. (1974). The Teacher as an enemy. In G. Spindler (Ed.), Educational and Cultural Process. Toward an Anthropology of Education (pp. 411-425). New York: Holt, Rinehart and Winston. 203.

Wolcott, H. (1985). On ethnographic intent. Educational Administration Quarterly, 21(3), 187-

Youdell, D. (2004). Engineering school markets, constituting schools and subjectivating students: the bureaucratic,institutional and classroom dimensions of educational triage. Journal of Education Policy, 19(4), 407-431. doi:10.1080/0268093042000227474 
